\title{
Estimativa de plastocrono em crambe
}

\author{
Estimating the plastochron in crambe
}

\section{Marcos Toebe ${ }^{\mathrm{I}^{*}}$ Sidinei José Lopes ${ }^{\mathrm{II}}$ Lindolfo Storck $^{\mathrm{II}}$ Tatiani Reis da Silveira ${ }^{\mathrm{I}}$ Marília Milani' Gabriele Casarotto ${ }^{\mathrm{I}}$}

\section{RESUMO}

Um parâmetro utilizado em modelos de simulação do desenvolvimento de espécies vegetais é o intervalo de tempo entre o aparecimento de dois nós sucessivos em uma haste, conhecido como plastocrono, com unidade de ${ }^{\circ} \mathrm{C}$ dia nó ${ }^{-1}$. $\mathrm{O}$ objetivo deste trabalho foi estimar o plastocrono em plantas de crambe (Crambe abyssinica Hochst) cultivadas em duas épocas de semeadura: 18/06/08 e 12/07/08. A cultivar usada foi a 'FMS Brilhante', com densidade ajustada para 100 plantas $\mathrm{m}^{-2}$. Em cada época, foram marcadas aleatoriamente 77 plantas, das quais se contou o número de nós a cada dois dias. O plastocrono foi estimado pelo inverso do coeficiente angular da regressão linear entre o número de nós acumulados na haste principal e a soma térmica acumulada após a emergência. O ciclo da cultura foi dividido em subperíodos, a fim de avaliar a demanda calórica em cada estádio de desenvolvimento. O plastocrono variou de 42,1 a $45,2^{\circ} \mathrm{C}$ dia nó ${ }^{-1}$ no subperíodo emergência até o início do florescimento e 28,3 a 47,8 $8^{\circ} \mathrm{C}$ dia nó ${ }^{-1}$ no subperíodo início do florescimento até o início da frutificação. Diferentes épocas de semeadura e subperíodos de desenvolvimento apresentam efeito sobre o plastocrono em crambe. Para a obtenção de estimativas seguras de plastocrono de crambe, a melhor subdivisão do ciclo é da emergência até o início do florescimento e do início do florescimento até o início da frutificação.

Palavras-chave: Crambe abyssinica Hochst, aparecimento de nós, desenvolvimento vegetal, soma térmica.

\section{ABSTRACT}

A parameter used in the development plants models simulation development is the time interval between the appearance of two successive nodes on a stem, known as plastochron, with units of ${ }^{\circ} \mathrm{C}$ day node $e^{-1}$. The objective of this study was to estimate the plastochron in crambe (Crambe abyssinica Hochst) plants grown in two sowing dates: 18/06/ 08 and 12/07/08. The cultivar used was the 'FMS Brilhante', with density adjusted to 100 plants $\mathrm{m}^{-2}$. In each period 77 plants were randomly selected, and the number of nodes were counted every two days. The plastochron was estimated by the inverse slope of the linear regression between the number of accumulated nodes on the main stem and accumulated thermal time after the emergence. The crop cycle was divided into subperiods in order to assess caloric demand for each development stage. The plastochron ranged from 42.1 to $45.2^{\circ} \mathrm{C}$ day node ${ }^{-1}$ during the subperiod emergence to early flowering and from 28.3 to $47.8^{\circ} \mathrm{C}$ day node $e^{-1}$ during the beginning of the flowering subperiod to the onset of fruiting. Different sowing dates and development subperiods have an effect on the plastochron in crambe. The best division of the cycle is the emergence to early flowering and early flowering to the onset of fruiting to obtain reliable estimates of plastochron crambe.

Key words: Crambe abyssinica Hochst, node appearance, plant development, thermal time.

\section{INTRODUÇÃO}

O crambe (Crambe abyssinica Hochst) pertence à família Brassicaceae, originária da região do Mediterrâneo e com relatos de ocorrência de algumas espécies na Etiópia (WEISS, 2000). É considerada cultura tolerante ao frio com potencial para produção industrial de biocombustíveis, pelo elevado potencial lubrificante e teor de óleo em sua composição, com valores entre 30 e $45 \%$ da semente.

Existem pesquisas em relação às características agronômicas da cultura (DAHNKE et

\footnotetext{
'Curso de Agronomia, Centro de Ciências Rurais (CCR), Universidade Federal de Santa Maria (UFSM), 97105-900, Santa Maria, RS, Brasil. E-mail: m.toebe@gmail.com.*Autor para correspondência.

"Departamento de Fitotecnia, CCR, UFSM, Santa Maria, RS, Brasil.
} 
al., 1992) e à determinação de temperatura base e de produtividade (KMEC et al., 1998). No entanto, no Brasil, não existem trabalhos recentes relacionados à fenologia de crambe, importantes na definição de: tratos culturais, época de semeadura, estimativa de data de colheita e manejo da espécie.

A quantificação do desenvolvimento vegetal é de grande importância nos estudos da biologia de espécies, pois está relacionada com: a diferenciação celular, a iniciação e o aparecimento de órgãos, estendendo-se até a senescência, sendo fundamental para fins de manejo das espécies vegetais (WILHELM \& McMASTER, 1995). Um exemplo de parâmetro de desenvolvimento vegetal é o número de nós acumulados (NN) na haste principal (FAGUNDES et al., 2008). Em leguminosas e algumas outras espécies como o crambe, em que cada nó origina uma folha, essa medida de desenvolvimento está relacionada com a expansão da área foliar, a qual tem relação com: a interceptação da radiação solar pelo dossel vegetal, a fotossíntese, o acúmulo de biomassa e a produtividade das culturas (XUE et al., 2004; DELLAI et al., 2005; STRECK et al., 2005; MARTINS et al., 2007).

O cálculo do NN na haste principal, nos modelos matemáticos, é realizado por meio do conceito de plastocrono, que é definido como o intervalo de tempo entre o aparecimento de dois nós sucessivos em uma planta (BAKER \& REDDY, 2001; STRECK et al., 2005). A unidade de tempo usada no cálculo de plastocrono é a soma térmica, com unidade em ${ }^{\circ} \mathrm{C}$ dia nó $^{-1}$.

A soma térmica é a medida de tempo biológico que leva em conta o efeito da temperatura nos processos fisiológicos da planta, sendo mais adequado e realista que o tempo expresso em dias, pois o efeito da temperatura é um dos principais fatores meteorológicos que governam o desenvolvimento de plantas, incluindo o aparecimento de nós e folhas (GILMORE \& ROGERS, 1958;ARNOLD, 1960; STRECK et al., 2008). A soma térmica diária é obtida pela temperatura média diária do ar subtraída da temperatura base (GILMORE \& ROGERS, 1958; ARNOLD, 1960). A temperatura base é definida como o valor da temperatura do ar abaixo do qual não ocorre desenvolvimento ou esse desenvolvimento é tão lento que, para fins de cálculo, pode ser considerado desprezível (McMASTER \& WILHELM, 1997).

A avaliação do desenvolvimento das culturas agrícolas em diferentes épocas de semeadura é importante, pois permite verificar a adaptação da cultura e as práticas de manejo mais adequadas (STRECK et al., 2007). Para diferentes genótipos de uma mesma espécie e para semeaduras em diferentes épocas de um mesmo genótipo, foram verificados diferentes valores de filocrono ou de plastocrono (STRECK et al., 2006; 2007; 2008b). Ainda, a adubação nitrogenada pode exercer influência na duração dos subperíodos de desenvolvimento e nas alterações de filocrono e plastocrono (FAGUNDES et al., 2007). Considerando esses fatos e as poucas informações sobre crambe no Brasil, o objetivo deste trabalho foi estimar o plastocrono em uma cultivar de crambe, em duas épocas de semeadura, nos subperíodos de desenvolvimento.

\section{MATERIAL E MÉTODOS}

O experimento foi conduzido na área experimental do Departamento de Fitotecnia da Universidade Federal de Santa Maria, RS (latitude: 2943'S, longitude: 5342'W e altitude: $95 \mathrm{~m}$ ). O clima da região é do tipo fundamental Cfa subtropical úmido, conforme classificação de Köppen. O solo do local é uma transição entre a Unidade de Mapeamento São Pedro (Argissolo Vermelho distrófico arênico) e a Unidade de Mapeamento Santa Maria (Alissolo Hipocrômico argilúvico típico) (STRECK et al., 2008a).

Foram realizadas duas épocas de semeadura em 18/06/08 (época 1) e 12/07/08 (época 2), e a emergência foi observada, respectivamente, nos dias 28/06/08 e 18/07/08. Foi utilizada a cultivar de crambe 'FMS Brilhante'. A adubação de base nas duas épocas consistiu de $35 \mathrm{~kg} \mathrm{ha}^{-1}$ de N, $140 \mathrm{~kg} \mathrm{ha}^{-1}$ de $\mathrm{P}_{2} \mathrm{O}_{5}$ e $140 \mathrm{~kg}$ $\mathrm{ha}^{-1}$ de $\mathrm{K}_{2} \mathrm{O}$. Na época 2, foi realizada adubação de cobertura, com uréia, totalizando $200 \mathrm{~kg} \mathrm{ha}^{-1}$ de $\mathrm{N}$, visando aumentar o índice de área foliar e a produção de biomassa. A densidade foi ajustada por meio de desbaste para 100 plantas $\mathrm{m}^{-2}$, com distância entre fileiras de $0,17 \mathrm{~m}$ e entre plantas de $0,0588 \mathrm{~m}$, e no momento da senescência a densidade foi de 104,4 e 96,5 plantas $\mathrm{m}^{-2}$, respectivamente, para as épocas 1 e 2 .

Em cada época, foram marcadas aleatoriamente, logo após a emergência, 77 plantas, das quais foi quantificado o número de nós visíveis na planta a cada dois dias até a senescência. Cada uma dessas plantas foi considerada uma repetição. Considerou-se um novo nó quando a folha associada a esse nó apresentava-se expandida, considerado o momento em que as bordas das folhas já se apresentavam planas, sem encurvamento. Foram medidos semanalmente o comprimento (C) e a largura (L) de todas as folhas de cada planta e utilizou-se a equação: $\mathrm{AF}=0,6603 *(\mathrm{C} * \mathrm{~L})$ (TOEBE et al., 2009) para a determinação da área foliar de cada planta. O índice de área foliar (IAF) foi determinado pela relação entre a área foliar de cada planta e o respectivo espaço de solo 
ocupado por ela $\left(\mathrm{m}^{2} \mathrm{~m}^{-2}\right)$. Avaliou-se semanalmente a altura de plantas $(\mathrm{cm})$ por meio de régua milimetrada, considerando a altura máxima da planta a partir da superfície do solo.

Os dados diários de temperatura mínima e máxima do ar foram coletados em estação meteorológica convencional, pertencente ao $8^{\circ}$ Distrito de Meteorologia do Instituto Nacional de Meteorologia, localizada a aproximadamente $30 \mathrm{~m}$ da área experimental. A soma térmica diária (STd, ${ }^{\circ} \mathrm{C}$ dia) foi calculada de acordo com ARNOLD (1960): STd=(Tmed - Tb). 1 dia, em que: Tmed é a temperatura média do ar, calculada pela média aritmética entre as temperaturas mínima e máxima diárias do ar; e Tb é a temperatura base para a cultura de crambe, de $2,5^{\circ} \mathrm{C}$ (KMEC et al., 1998). A soma térmica acumulada (STa, ${ }^{\circ} \mathrm{C}$ dia) a partir do dia de emergência foi calculada por: STa $=\Sigma \mathrm{STd}$.

Para cada época de cultivo, obteve-se a regressão linear entre o número de nós acumulados (NN) na planta e a soma térmica acumulada (STa) a partir da emergência . O plastocrono $\left({ }^{\circ} \mathrm{C}\right.$ dia nó $\left.{ }^{-1}\right)$ foi considerado como sendo o inverso do coeficiente angular da regressão linear entre NN e STa (KLEPPER et al., 1982; BAKER \& REDDY, 2001, SINCLAIR et al., 2005). A fim de determinar o plastocrono nos diferentes subperíodos, foram gerados, para as duas épocas, modelos de regressão considerando os seguintes subperíodos: emergência até o início do florescimento (EM-FL), emergência até o início da frutificação (EMFRUT), emergência até a senescência (EM-SEN), início do florescimento até o início da frutificação (FL-FRUT) e início da frutificação até a senescência (FRUT-SEN). Na época 2, o subperíodo de emergência até início do florescimento foi dividido em emergência até a adubação nitrogenada (EM-AN) e após adubação nitrogenada até o início do florescimento (AN-FL). O início do florescimento foi definido como sendo a data em que $50 \%$ das plantas avaliadas apresentassem pelo menos uma inflorescência visível. O início da frutificação foi considerado como sendo a data em que $50 \%$ das plantas avaliadas apresentassem pelo menos um fruto em formação. A senescência foi considerada como sendo o momento em que $50 \%$ ou mais das plantas avaliadas não apresentavam mais folhas.

As médias de nós por subperíodos foram comparadas entre as épocas 1 e 2 pelo teste t, em $1 \%$ de probabilidade de erro. As equações de estimativa de plastocrono foram obtidas por meio do aplicativo Office Excel.

\section{RESULTADOS E DISCUSSÃO}

A soma térmica diária (STd) média no subperíodo emergência até o início do florescimento foi de 13,6 e $13,3^{\circ} \mathrm{C}$ dia, nas épocas 1 e 2 , respectivamente. Embora esses valores médios sejam próximos, a menor STd, na época 2, contribuiu para a maior duração do subperíodo em dias, e o início do florescimento ocorreu aos 42 e 50 dias após a emergência para as épocas 1 e 2, respectivamente (Figura 1A). No subperíodo compreendido entre o início da frutificação e a senescência, foram verificados baixos valores de STd na época 1 , com valor mínimo de $5,25^{\circ} \mathrm{C}$ dia. Na época 2, o valor mínimo de STd nesse mesmo subperíodo foi de $10,65^{\circ} \mathrm{C}$ dia, evidenciando condições térmicas mais adequadas ao desenvolvimento da cultura (Figura 1A).

O comportamento da estatura média de plantas foi similar nas duas épocas, e os valores máximos foram de 66,5 e 72,2cm, respectivamente, nas épocas 1 e 2 (Figura 1 B). Os valores máximos de índice de área foliar (IAF) médio foram de 1,57 e 2,06 nas épocas 1 e 2, respectivamente (Figura $1 \mathrm{C}$ ). O baixo valor do IAF observado na época 1 motivou a suplementação da adubação nitrogenada na época 2, visto que baixos valores de IAF indicam a dificuldade da cultura em cobrir completamente a superfície do solo, dificultando o manejo conservacionista do solo. Em experimentos de crambe, conduzidos durante três anos, KMEC et al. (1998) encontraram valores de IAF máximos variando de 1,8 a 2,7. Com a adubação nitrogenada, na época 2, foi possível observar o incremento no IAF e a redução da velocidade de senescência das folhas de crambe, comportamento similar ao observado na cultura do girassol (FAGUNDES et al., 2007)

Diferentes valores de plastocrono foram obtidos para as duas épocas, o que também foi verificado em outras culturas, como na cultura do arroz, em que o filocrono é mais influenciado pelas épocas de semeadura em relação a diferentes genótipos (STRECK et al., 2007). Na época 1, no subperíodo EM-FL, foi verificada a demanda de $45,2^{\circ} \mathrm{C}$ dia nó ${ }^{-1}$ (Figura 2A) e na época 2 a demanda foi de $42,1^{\circ} \mathrm{C}$ dia nó ${ }^{-1}$ (Figura 2B). A menor demanda calórica na época 2 pode ser atribuída ao efeito do nitrogênio, que atuaria no aumento da taxa de emissão de novos nós. Essa redução de aproximadamente $3^{\circ} \mathrm{C}$ dia nó ${ }^{-1}$ corrobora o estudo realizado com a cultura do girassol de vaso, em que foi verificada redução de 25,4 para $22,0^{\circ} \mathrm{C}$ dia folha ${ }^{-1}$, quando realizada adubação de cobertura com nitrogênio (FAGUNDES et al., 2007). Na época 2, por meio da divisão do subperíodo EM-FL, é possível identificar a semelhança dos valores de plastocrono, 


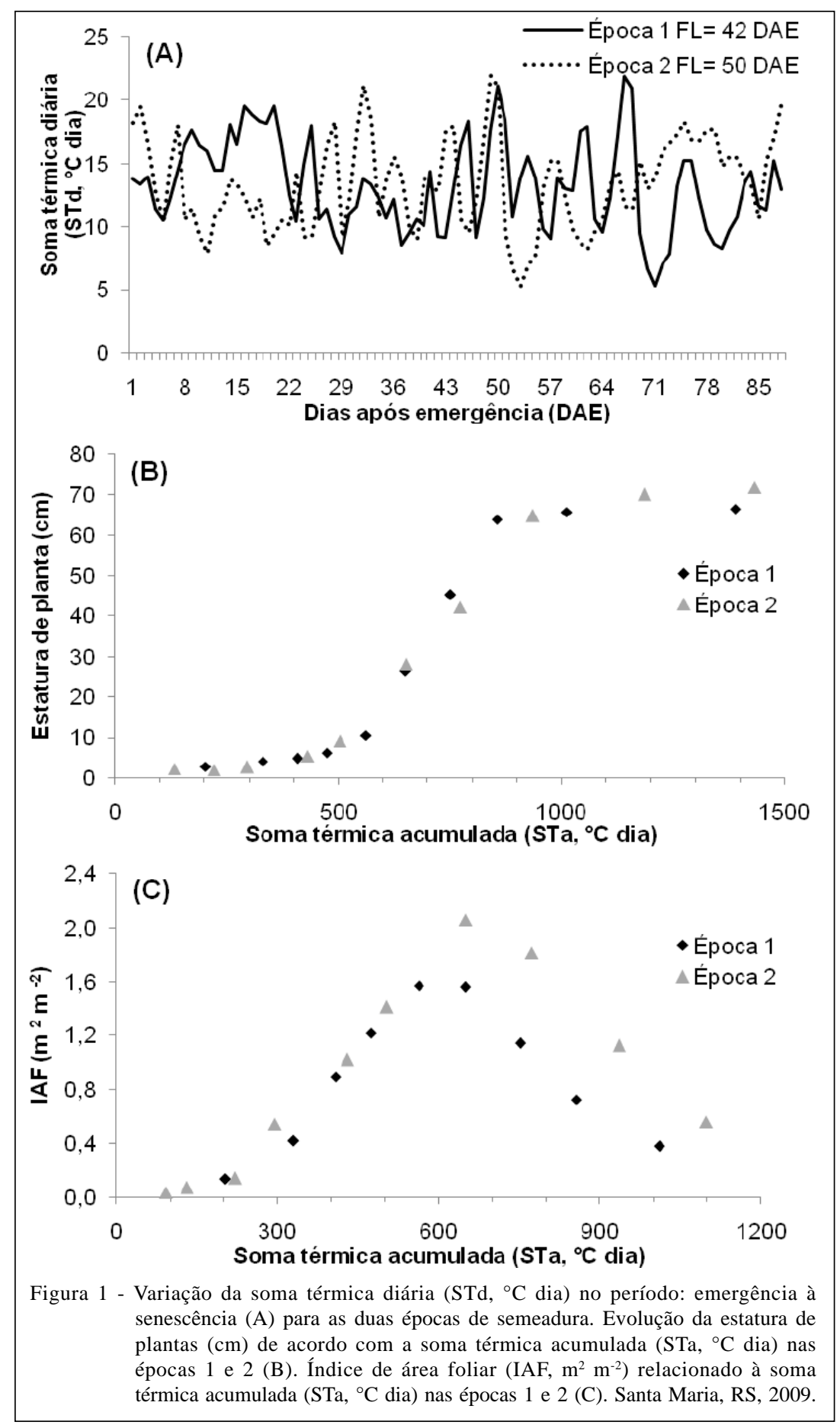

antes da adubação de nitrogênio, com os valores observados na época 1. Após a adubação, nota-se uma redução de aproximadamente $6^{\circ} \mathrm{C}$ dia nó ${ }^{-1}$ (Tabela 1 ).

Mesmo que a época 2 tenha apresentado maior velocidade de emissão de nós no subperíodo EM-FL, a duração foi maior em relação à época 1 (Tabela 1). Assim, o número final de nós foi de 11,1 e 13,4, respectivamente, para as épocas 1 e 2 , o que permite inferir que a adubação nitrogenada, bem como a data de semeadura da época 2, retardaram o florescimento da cultura, apresentando um maior IAF no momento do florescimento (Figura $1 \mathrm{C}$ ). Quanto à soma térmica acumulada no subperíodo EM-FL, a diferença de 571,6 para $671,5^{\circ} \mathrm{C}$ entre as épocas é semelhante à variação 

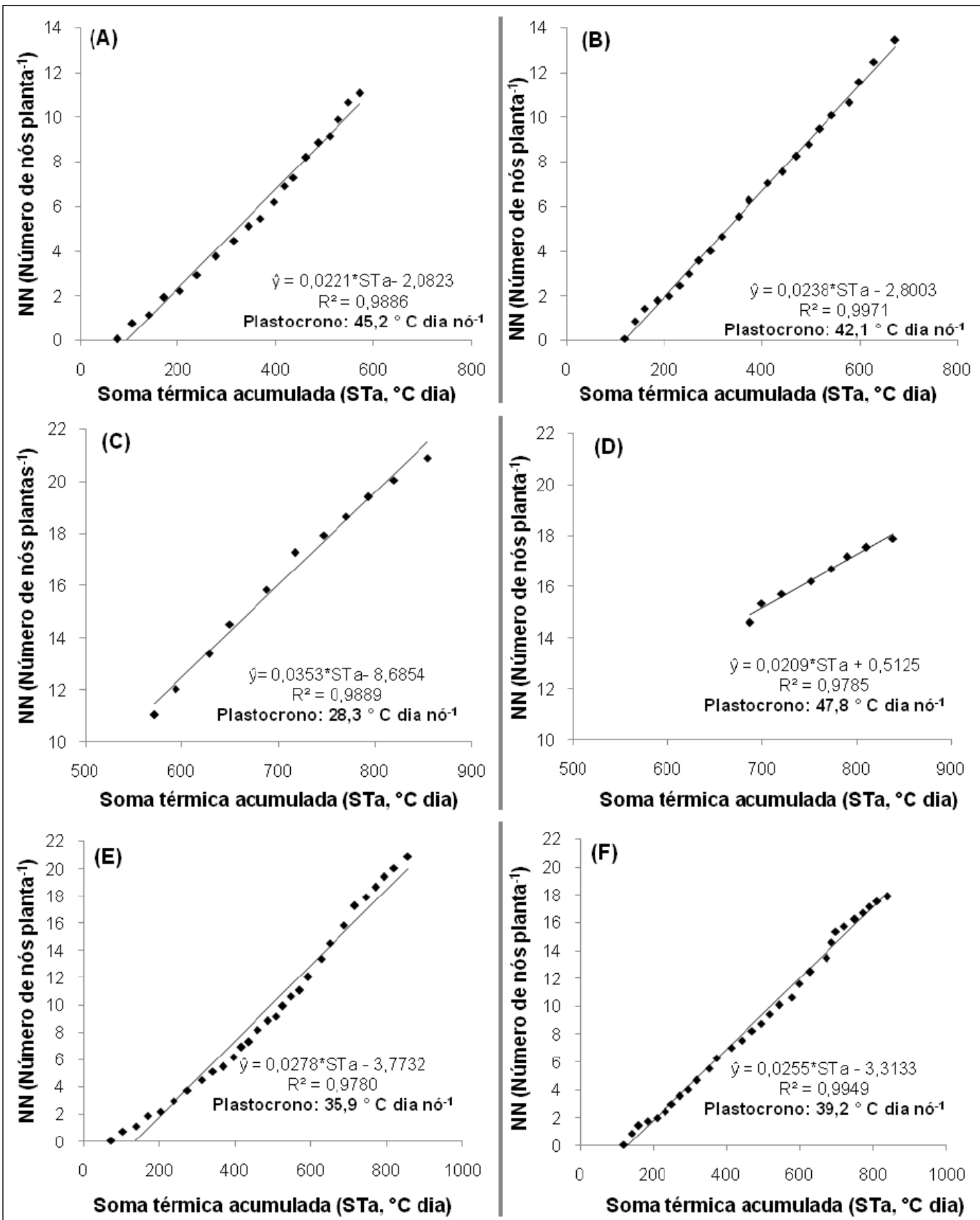

Figura 2 - Relação entre número de nós acumulados na haste principal da planta (NN, nós planta-1) e soma térmica acumulada (STa, ${ }^{\circ} \mathrm{C}$ dia), utilizada para estimativa do plastocrono nos períodos: emergência-florescimento (A e B), florescimento-frutificação (C e D) e emergência-frutificação (E e F), respectivamente, para as épocas 1 e 2. Santa Maria, RS, 2009.

encontrada por KMEC et al. (1998), que verificaram variações de 594 a $700^{\circ} \mathrm{C}$ de STa para o surgimento do botão floral.

No subperíodo FL-FRUT, nas plantas da época 1 , houve aumento da velocidade de emissão de nós, em relação ao subperíodo EM-FL (Figura 2A, C). Na época 2, observou-se menor emissão de nós no subperíodo FL-FRUT, parte pela redução do efeito do nitrogênio e também pelo maior subperíodo EM-FL e maior número de nós no início de florescimento (Figura 
Tabela 1 - Número de dias de observações (Obs), soma térmica acumulada (STa, ${ }^{\circ} \mathrm{C}$ dia), número final de nós ( $\mathrm{N}^{\circ}$ nós), equação estimada por meio da correlação entre $\mathrm{NN}$ e STa, valores do coeficiente de determinação $\left(\mathrm{R}^{2}\right)$, raiz do quadrado médio do erro (DP) e plastocrono (PLAST. em ${ }^{\circ} \mathrm{C}$ dia nó ${ }^{-1}$ ). Santa Maria, RS, 2009.

\begin{tabular}{llllcccc}
\hline \multicolumn{1}{c}{ Período } & Obs & STa & $\mathrm{N}^{\circ}$ nós & Equação Estimada & $\mathrm{R}^{2}$ & DP & PLAST. \\
\hline EM-FL & 19 & 571,6 & $11,1^{* *}$ & $\mathrm{y}=-2,082258+0,022118^{*} \mathrm{STa}$ & 0,989 & 0,384 & 45,2 \\
EM-FRUT & 29 & 854,9 & $19,9^{* *}$ & $\mathrm{y}=-3,773194+0,027839^{*} \mathrm{STa}$ & 0,978 & 0,970 & 35,9 \\
EM-SE & 43 & 1165,3 & $21,8^{* *}$ & $\mathrm{y}=-1,992409+0,023621^{*} \mathrm{STa}$ & 0,957 & 1,624 & 42,3 \\
FL-FRUT & 11 & 283,3 & $8,9^{* *}$ & $\mathrm{y}=-8,685359+0,035304 * \mathrm{STa}$ & 0,989 & 0,371 & 28,3 \\
FRUT-SEN & 14 & 310,4 & $1,9^{\text {ns }}$ & $\mathrm{y}=18,320677+0,003064 * \mathrm{STa}$ & 0,914 & 0,095 & 326,4 \\
& & & & Época 2 & & \\
EM-FL & 22 & 671,5 & 13,4 & $\mathrm{y}=-2,800281+0,023763^{*} \mathrm{STa}$ & 0,997 & 0,225 & 42,1 \\
EM-FRUT & 30 & 838,2 & 17,9 & $\mathrm{y}=-3,313344+0,025487 * \mathrm{STa}$ & 0,995 & 0,420 & 39,2 \\
EM-SE & 41 & 1175,8 & 19,0 & $\mathrm{y}=-1,338802+0,020710^{*} \mathrm{STa}$ & 0,950 & 1,514 & 48,3 \\
FL-FRUT & 8 & 166,7 & 4,4 & $\mathrm{y}=0,512541+0,020936 * \mathrm{STa}$ & 0,978 & 0,180 & 47,8 \\
FRUT-SEN & 12 & 337,6 & 1,2 & $\mathrm{y}=16,178069+0,002636 * \mathrm{STa}$ & 0,588 & 0,262 & 379,3 \\
EM-AN & 10 & 317,6 & 4,7 & $\mathrm{y}=-2,269909+0,021291 * \mathrm{STa}$ & 0,983 & 0,201 & 47,0 \\
AN-FL & 12 & 353,9 & 8,7 & $\mathrm{y}=-3,106254+0,024383 * \mathrm{STa}$ & 0,994 & 0,200 & 41,0 \\
\hline
\end{tabular}

**Diferença significativa entre épocas pelo teste t, em nível de 1\% de probabilidade de erro; ${ }^{\text {ns }}$ não significativo; EM=emergência; FL=início do florescimento; FRUT= início da frutificação; SEN=senescência; AN=adubação nitrogenada.

2 B, D). O subperíodo FL-FRUT foi menor na época 2, provavelmente, pelo aumento do comprimento do dia e também pela maior STd (Figura 1A). Considerando o subperíodo EM-FRUT (Figura 2 E, F), a época 1 apresentou maior velocidade de emissão de nós, impulsionada pela rápida emissão após o início do florescimento.

Os menores valores da raiz do quadrado médio do erro (DP) (Tabela 1) foram obtidos nos subperíodos EM-FL e FL-FRUT para as duas épocas, podendo ser considerados os de maior precisão na obtenção dos dados referentes ao desenvolvimento de crambe. Mesmo com altos valores de coeficiente de determinação e valores de DP menores do que 1, os modelos matemáticos que consideram o subperíodo EM-FRUT são menos adequados, em razão da variação do plastocrono entre os subperíodos, ocorrido nessas duas épocas. Para fins de cálculo do plastocrono, o período FRUT-SEN pode ser desconsiderado pela insignificante emissão de nós (Tabela 1).

\section{CONCLUSÕES}

Diferentes épocas de semeadura e subperíodos de desenvolvimento apresentam efeito sobre o plastocrono em crambe, com variações de 28,3 a $47,8^{\circ} \mathrm{C}$ dia nó ${ }^{-1}$. Para a obtenção de estimativas seguras de plastocrono em crambe, a melhor subdivisão do seu ciclo é da emergência até o início do florescimento e do início do florescimento até o início da frutificação.

\section{REFERÊNCIAS}

ARNOLD, C.Y. Maximum-minimum temperatures as a basis for computing heat units. Proceedings of the American Society for Horticultural Sciences, v.76, p.682-692, 1960.

BAKER, J.T.; REDDY, V.R. Temperature effects on phenological development and yield of muskmelon. Annals of Botany, v.87, p.605-613, 2001. Disponível em: <http:// aob.oxfordjournals.org/cgi/reprint/87/5/605.pdf>. Acesso em: 06 set. 2009. doi: 10.1006/anbo.2001.1381.

DAHNKE, W.C. et al. Fertilizing mustard, rapessed, canola and crambe. Fargo, ND: NDSU, 1992. (Extension Publication SF-718).

DELLAI, J. et al. Filocrono em diferentes densidades de plantas de batata. Ciência Rural, v.35, p.1269-1274, 2005. Disponível em: $<$ http://www.scielo.br/scielo.php?script=sci_arttext\&pid=S010384782005000600007\&lng=pt\&nrm=iso $>$. Acesso em: 17 nov. 2009. doi: 10.1590/S0103-84782005000600007.

FAGUNDES, J.D. et al. Crescimento, desenvolvimento e retardamento da senescência foliar em girassol de vaso (Helianthus annuus L.): fontes e doses de nitrogênio. Ciência Rural, v.37, p.987-993, 2007. Disponível em: <http:// www.scielo.br/scielo.php?script $=$ sci_arttext\&pid $=$ S010384782007000400011\&lng=e\&nrm=iso $>$. Acesso em: 06 set. 2009. doi: 10.1590/S0103-84782007000400011 .

FAGUNDES, J.D. et al. Temperatura base, plastocrono e número final de nós no malmequer-do-campo. Ciência Rural, v.38, p.2471-2477, 2008. Disponível em: <http://www.scielo.br/ scielo.php? script=s ci_art text\&pid=S 0103 84782008000900009\&lng=pt\&nrm=iso>. Acesso em: 17 nov. 2009. doi: 10.1590/S0103-84782008005000021. 
GILMORE Jr., E.C.; ROGERS, J.S. Heat units as a method of measuring maturity in corn. Agronomy Journal, v.50, p.611615, 1958.

KLEPPER, B. et al. Quantitative characterization of vegetative development in small cereal grains. Agronomy Journal, v.7, p.780-792, 1982

KMEC, P. et al. Growth analysis of crambe. Crop Science, v.38, p.108-112, 1998.

MARTINS, F.B. et al. Estimativa da temperatura-base para emissão de folhas e do filocrono em duas espécies de eucalipto na fase de muda. Revista Árvore, v.31, p.373-381, 2007. Disponível em: $<$ http://www.scielo.br/scielo.php?script=sci arttext\&pid=S010067622007000300002\&lng=pt\&nrm=iso $>$. Acesso em: 17 nov. 2009. doi: 10.1590/S0100-67622007000300002..

McMASTER, G.S.; WILHELM, W.W. Growing degree-days: one equation, two interpretations. Agricultural and Forest Meteorology, v.87, p.291-300, 1997. Disponível em: < h t t p : / / d i g i t a l c o m mo n s.un l. ed u / c g i / viewcontent.cgi ?article $=1086 \&$ context $=$ usdaarsfacpub $>$. Acesso em: 06 set. 2009.

SINCLAIR, T.R. Leaf area development in field grown soybean. Agronomy journal, v.76, p.141-146, 1984.

SINCLAIR, T.R. et al. Comparison of vegetative development in soybean cultivars for low-latitude environments. Field Crops Research, v.92, p.53-59, 2005.

STRECK, E.V. et al. Solos do Rio Grande do Sul. 2.ed. Porto Alegre: Emater/RS-ASCAR, 2008a. 222p.

STRECK, N.A. et al. Estimativa do plastocrono em meloeiro (Cucumis melo L.) cultivado em estufa plástica em diferentes épocas do ano. Ciência Rural, v.35, p.1275-1280, 2005. Disponível em: $<$ http://www.scielo.br/scielo.php?script=sci_arttext\&pid=S0103- 84782005000600008\&lng=pt\&nrm=iso>. Acesso em: 17 nov. 2009. doi: 10.1590/S0103-84782005000600008.

STRECK, N.A. et al. Duração do ciclo de desenvolvimento de cultivares de arroz em função da emissão de folhas no colmo principal. Ciência Rural, v.36, p.1086-1093, 2006. Disponível em: <http:/ /www.scielo.br/scielo.php?script=sci_arttext\&pid=S0103$84782006000400007 \& \operatorname{lng}=$ pt\&nrm=iso $>$. Acesso em: 17 nov. 2009. doi: 10.1590/S0103-84782006000400007.

STRECK. N.A. et al. Filocrono de genótipos de arroz irrigado em função de época de semeadura. Ciência Rural, v.37, p.323-329, 2007. Disponível em: <http://www.scielo.br/ scielo.php ? s c ri p t = sci_art text \& pid = S 0103 $84782007000200005 \& \operatorname{lng}=p t \& n r m=i s o>$. Acesso em: 17 nov. 2009. doi: 10.1590/S0103-84782007000200005

STRECK, N.A. et al. Estimativa do plastocrono em cultivares de soja. Bragantia, v.67, p.67-73, 2008b. Disponível em: <http://www.scielo.br/scielo.php?script=sci_arttext\&pid=S0006$87052008000100008 \& \operatorname{lng}=$ pt\&nrm=iso $>$. Acesso em: 17 nov. 2009. doi: 10.1590/S0006-87052008000100008.

TOEBE, M. et al. Modelos para estimativa da área foliar de Crambe abyssinica por fotos digitais. In: REUNIÃO ANUAL DA REGIÃO BRASILEIRA DA SOCIEDADE INTERNACIONAL DE BIOMETRIA, 54.; SIMPÓSIO DE ESTATÍSTICA APLICADA À EXPERIMENTAÇÃO AGRONÔMICA, 13., 2009, São Carlos. Anais... São Carlos: Universidade Federal de São Carlos, 2009. V.1

WILHELM, W.W.; McMASTER, G.S. Importance of the phyllochron in studying development and growth in grasses. Crop Science, v.35, p.1-3, 1995.

WEISS, E. A. Oilseed crops. London: Blackwell Science, 2000. 364p.

XUE, Q. et al. Predicting leaf appearance in field-grown winter wheat: evaluating linear and non-linear models. Ecological Modelling, v.175, p.261-270, 2004 